\title{
Intoxicação experimental com frutos de Melia azedarach (Meliaceae) em suínos ${ }^{1}$
}

\author{
Maria del Carmen Méndez ${ }^{2 *}$, Fabiana Elias ${ }^{3}$, Franklin Riet-Correa ${ }^{4}$, Eduardo Juan \\ Gimeno $^{5}$ e Enrique Leo Portiansky ${ }^{5}$
}

\begin{abstract}
Méndez M.C., Elias F., Riet-Correa F., Gimeno E.J. \& Portiansky E. 2006. [Experimental poisoning by fruits of Melia azedarach (Meliaceae) in pigs.] Intoxicação experimental com frutos de Melia azedarach (Meliaceae) em suínos. Pesquisa Veterinária Brasileira 26(1):26-30. Departamento de Clínicas Veterinárias, Faculdade de Veterinária, Universidade Federal de Pelotas, 96010-900 Pelotas, RS, Brazil. E-mail: nane@ufpel.tche.br

Mature ground fruits of Melia azedarach were administered mixed with the food to 8 pigs at single doses of $5-20 \mathrm{~g} / \mathrm{kg}$. Transient diarrhea was the only sign observed in the 2 pigs that ingested $5 \mathrm{~g} / \mathrm{kg}$. Clinical signs observed in the pigs that ingested 10,15 and $20 \mathrm{~g} / \mathrm{kg}$ were incoordination, muscular tremors, difficulty to stand or to get up, sternal decubitus and hypothermia. The 2 pigs that ingested $20 \mathrm{~g} / \mathrm{kg}$ died. The gross lesions were congestion and necrosis of the small intestine and the stomach mucosa. The microscopic alterations were characterised by necrosis of the lymphatic tissues and skeletal muscles, congestion and necrosis of the stomach and small intestine, and moderate degenerative alterations of liver and kidneys. The serum levels of AST and CPK increased after the ingestion of the toxic fruits. High values of CPK were observed in the succumbing animals due to the muscle necrosis. These results demonstrate that the ingestion of high doses of Melia azedarach fruits by pigs may induce clinical signs of poisoning and cause lesions in several organs and even death.
\end{abstract}

INDEX TERMS: Poisonous plants, plant poisoning, Melia azedarach, Meliaceae, swine.

RESUMO.- Frutos maduros, triturados, de Melia azedarach foram administrados misturados à ração a 8 suínos em doses únicas de $5-20 \mathrm{~g} / \mathrm{kg}$. Diarréia passageira foi o único sinal nos 2 suínos que consumiram $5 \mathrm{~g} / \mathrm{kg}$. Os sinais clínicos observados nos animais que ingeriram doses únicas de 10,15 e $20 \mathrm{~g} / \mathrm{kg}$ caracterizaram-se por incoordenação, tremores musculares, dificuldade para manter-se de pé, relutância para levantar-se, decúbito esternal e hipotermia. Os 2 suínos que ingeriram $20 \mathrm{~g} / \mathrm{kg}$ morreram. Macroscopicamente observou-se congestão do intestino

\footnotetext{
${ }^{1}$ Recebido em 24 de maio de 2005.

Aceito para publicação em 21 de outubro de 2005.

Parte da Dissertação de Doutorado em Ciências Veterinárias do primeiro autor, Universidade Nacional de La Plata, Argentina.

${ }^{2}$ Depto Clínicas Veterinárias, Faculdade de Medicina Veterinária, Universidade Federal de Pelotas (UFPel), Pelotas, RS 96010-900, Brasil. *Autor para correspondência. E-mail: nane@ufpel.tche.br

3 Pós-graduanda em Medicina Veterinária, UFPel.

${ }^{4}$ Centro de Saúde e Tecnologia Rural, Universidade Federal de Campina Grande, Patos, PB 58700-000, Brasil. E-mail: riet@cstr.ufcg.edu.br

${ }^{5}$ Depto Patologia, Facultad de Ciencias Veterinarias, P.O. Box 296, 1900 La Plata, Argentina.
}

delgado e da mucosa do estômago. As alterações histológicas caracterizaram-se por discreta a moderada necrose do tecido linfático, necrose dos músculos esqueléticos, congestão e necrose da mucosa gástrica e do intestino delgado e alterações degenerativas moderadas no fígado e rim. Os níveis séricos de AST e CPK aumentaram após a ingestão do tóxico. Observaramse valores altos de CPK nos animais que morreram, evidenciando a mionecrose. Os resultados obtidos demonstram que, para suínos, a ingestão de altas doses de frutos de Melia azedarach induz ao aparecimento de sinais clínicos da intoxicação e causa lesões em diversos órgãos, inclusive a morte.

TERMOS DE INDEXAÇÃO: Plantas tóxicas, intoxicação por planta, Melia azedarach, Meliaceae, suínos.

\section{INTRODUÇÃO}

Melia azedarach, com nome vulgar de cinamomo, é uma árvore ornamental de crescimento rápido e de distribuição mundial (Kingsbury 1964). É nativa originalmente de Pérsia, Índia e China e atualmente aclimatada em diversas regiões como África, América do Sul, Austrália, Nova Zelândia, Indonésia, Japão, Sul dos Estados Unidos, Hawai e Bermuda. Atinge 6-12m e algumas vari- 
edades podem alcançar mais de 20m de altura (Hurst 1942, Kingsbury 1964, Oelrichs et al.1985, Hare et al. 1997). No Brasil, a planta está amplamente distribuída nas regiões subtropicais.

A intoxicação por $M$. azedarach tem sido mencionada no homem e em animais domésticos em conseqüência da ingestão de folhas ou de frutos caídos. (Kingsbury 1964, Everist 1974, Oelrichs et al. 1985). A toxicidade das plantas pode variar devido a fatores ambientais como área geográfica, clima, condições de crescimento da planta (Hurst 1942) e, em algumas plantas, pode estar totalmente ausente (Oelrichs et al. 1983, 1985).

Os suínos são os animais mais afetados pela intoxicação; porém os bovinos, ovinos, caprinos e aves, também podem ser afetados (Everist 1974, Oelrichs et al. 1985, Méndez et al. 2002a,b, Méndez 2003). A intoxicação em suínos ocorre geralmente devido à ingestão de frutos maduros, quando estes caem ao solo, podendo ocorrer, também, pela ingestão de folhas e frutos de galhos que estão ao seu alcance (Everist 1974, Kwatra et al. 1974).

No Rio Grande do Sul, observam-se casos de intoxicação por M. azedarach em suínos que ingerem os frutos que caem das árvores (Timm \& Riet-Correa 1997).

Experimentalmente a dose tóxica para suínos é de aproximadamente $0,5 \%$ do peso do animal (Hurst 1942, Kingsbury 1964). Nesta espécie, os sinais clínicos aparecem rapidamente, em 2-4 horas e descrevem-se dois quadros clínicos: a) náuseas, vômitos e constipação ou diarréia hemorrágica; ou b) signos nervosos de excitação ou depressão, dispnéia e ritmo cardíaco débil. A morte, geralmente, ocorre dentro de 24 horas; mas ocasionalmente pode demorar alguns dias e animais menos afetados podem recuperar-se lentamente (Kingsbury 1964, Oelrichs et al. 1985, Hare 1998). As lesões de necropsia não são específicas. Os achados mais comuns são congestão hepática e renal severas e difusas (Hothi et al. 1976). Nos casos mais agudos observa-se somente congestão gastrintestinal (Kingsbury 1964, Oelrichs et al. 1985). Os achados microscópicos mais característicos encontram-se principalmente no intestino delgado. Observam-se também cilindros hialinos nos túbulos renais e necrose de linfócitos nos gânglios linfáticos e no baço (Kwatra et al. 1974, Oelrichs et al. 1985)
O objetivo deste trabalho foi estudar experimentalmente a intoxicação por frutos de M. azedarach em suínos, principalmente no referente às alterações patológicas, incluindo as lesões musculares, que não têm sido adequadamente descritas.

\section{MATERIAL E MÉTODOS}

Frutos maduros de Melia azedarach L. (fam.Meliaceae) (Fig.1) foram recolhidos de árvores localizadas na Faculdade de Veterinária, no Campus Universitário da Universidade Federal de Pelotas, município de Capão do Leão, Rio Grande do Sul.

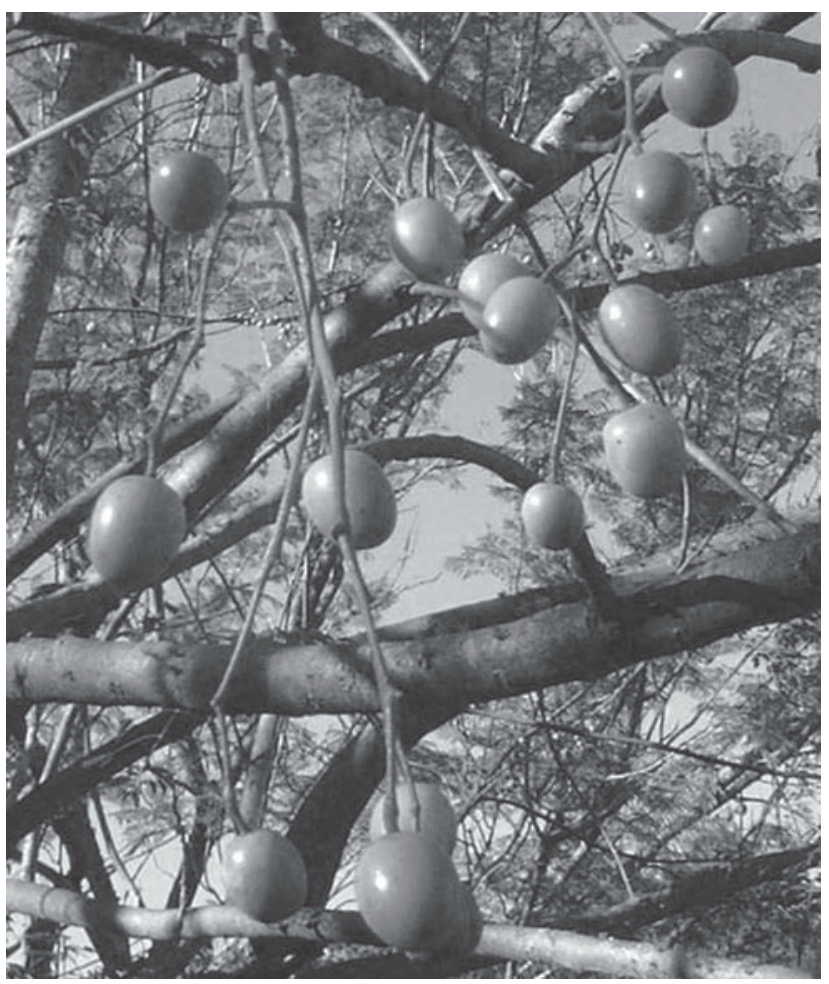

Fig. 1. Frutos de Melia azeradarach, município de Pelotas, Rio Grande do Sul.

Quadro 1. Peso corporal, doses de frutos de Melia azedarach administradas, sinais clínicos observados e evolução da intoxicação experimental em suínos

\begin{tabular}{|c|c|c|c|c|c|c|c|c|c|c|}
\hline \multirow{2}{*}{$\begin{array}{c}\text { Suíno } \\
n^{\circ}\end{array}$} & \multirow{2}{*}{$\begin{array}{c}\text { Peso } \\
\mathrm{kg}\end{array}$} & \multirow{2}{*}{$\begin{array}{c}\text { Doses } \\
\mathrm{g} / \mathrm{kg}\end{array}$} & \multicolumn{7}{|c|}{ Sinais clínicos } & \multirow[t]{2}{*}{ Evolução } \\
\hline & & & Diarréia & $\begin{array}{c}\text { Tremores } \\
\text { musculares }\end{array}$ & Incoordinação & Hipotermia & $\begin{array}{c}\text { Decúbito } \\
\text { esternal }\end{array}$ & Início & Duração & \\
\hline 1 & 8 & 5 & $+^{a}$ & - & - & - & - & $24 h$ & $18-24 h^{b}$ & Sobreviveu \\
\hline 2 & 10 & 5 & + & - & - & - & - & $24 h$ & $18-24 \mathrm{~h}$ & Sobreviveu \\
\hline 3 & 16 & 10 & - & + & + & + & + & $24 h$ & $18-24 \mathrm{~h}$ & Sobreviveu \\
\hline 4 & 22 & 10 & - & + & + & + & + & $3 \mathrm{~h}$ & $18-24 \mathrm{~h}$ & Sobreviveu \\
\hline 5 & 26 & 15 & - & - & - & - & - & $5 \mathrm{~h}$ & $18-24 \mathrm{~h}$ & Sobreviveu \\
\hline 6 & 8 & 15 & - & + & + & + & + & $24 h$ & $18-24 \mathrm{~h}$ & Sobreviveu \\
\hline 7 & 18 & 20 & - & + & + & + & + & $2 \mathrm{~h}$ & $1 \mathrm{~h}$ & Morreu \\
\hline 8 & 8 & 20 & - & + & + & + & + & $2 \mathrm{~h}$ & $3 \mathrm{~h}$ & Morreu \\
\hline 9 & 25 & 0 & - & - & - & - & - & - & - & Sobreviveu \\
\hline 10 & 20 & 0 & - & - & - & - & - & - & - & Sobreviveu \\
\hline
\end{tabular}

a + Presença do sinal clínico, - sua ausência.

b Como os animais não foram observados durante a noite não foi determinada à duração exata dos sinais clínicos. 
Os frutos maduros triturados foram misturados na ração e administrados em doses de 5, 10, 15 e $20 \mathrm{~g}$ por $\mathrm{kg}$ de peso do animal $(\mathrm{g} / \mathrm{kg})$. Foram utilizados 8 suínos machos Landrace de 60-120 dias de idade, pesando entre 8 e $26 \mathrm{~kg}$. Os animais foram mantidos em jejum por um período de 48 horas antes de iniciar o experimento. Dois suínos controles permaneceram juntos nas mesmas condições, recebendo a mesma ração comercial.

No Quadro 1 apresentam-se o peso dos suínos e as doses de frutos de cinamomo administradas.

Amostras de sangue por punção da veia cava foram extraídas de todos os animais, para determinação das enzimas aspartato aminotransferase (AST) e creatinina fosfoquinase (CPK). As amostras foram retiradas 24 horas antes de iniciar o experimento e posteriormente a cada 24 horas após a ingestão dos frutos, durante 3 dias. Nos suínos controles foi aplicado o mesmo protocolo para obter um valor médio da atividade enzimática durante o período do experimento.

Nos animais que morreram se realizou necropsia coletando amostras de diversos tecidos incluindo pulmão, coração, fígado, rim, linfonodos mesentéricos, estômago, intestino delgado e grosso, baço, sistema nervoso central e músculos esqueléticos (serrato, largo dorsal, semitendinoso, semimembranoso, psoas, intercostais, bíceps, triceps e diafragma). Os materiais foram fixados em formol a $10 \%$ e embebidos em parafina, cortados em seç̧ões de $6 \mathrm{~mm}$ e corados pela hematoxilinaeosina (HE), para o estudo histológico. Antes de serem fixados em formol as amostras de músculos esqueléticos foram mergulhadas em solução salina durante $40-50$ minutos e posteriormente refrigeradas a $4^{\circ} \mathrm{C}$ durante 12 horas; desta forma, para se evitar a formação de artefatos produzidos pela rigidez cadavérica.

Do Suíno 8, amostras de músculos esqueléticos foram processadas para o estudo por microscopia eletrônica. As amostras foram fixadas em solução de glutaraldeído a $2 \%$ e paraformaldeído a $2 \%$ em tampão cacodilato de sódio $(0,03 \mathrm{M}, \mathrm{pH} 7,4)$, pós-fixados em tretóxido de ósmio a 1\% e embebidos em Epon 812. Os cortes semifinos foram corados com azul de metileno. As áreas selecionadas foram cortadas com navalha de diamante e os cortes, contrastados com acetato de uranilo e citrato de chumbo, foram observados em microscópio eletrônico de transmissão.

Apesar do número de animais utilizados por cada dose ser baixo, realizou-se um estudo estatístico de correlação entre as doses de frutos administradas e os valores enzimáticos, assim como também um estudo de variância entre os valores obtidos para cada tempo de observação, as doses de frutos e as enzimas estudadas.

A correlação de $r>0,5$ (valores absolutos) foi considerada significativa. Para a variância, valor de $p<0,05$ foi considerado significativo.

\section{RESULTADOS}

Os suínos que ingeriram doses de $5 \mathrm{~g} / \mathrm{kg}$ de frutos apresentaram diarréia discreta e recuperaram-se rapidamente. Nos suínos que receberam doses de 10 e $20 \mathrm{~g} / \mathrm{kg}$ e, em um animal que recebeu $15 \mathrm{~g} / \mathrm{kg}$ de frutos (Suíno 6) os sinais clínicos caracterizaram-se por incoordinação, tremores musculares, gemidos e gritos, dificuldade para manter-se de pé, relutância para levantar-se, decúbito esternal e hipotermia. O tempo decorrido entre a ingestão e o aparecimento dos sinais clínicos foi de 2-24 horas. A evolução nos suínos que morreram foi de 1-3 horas e nos que sobreviveram de 18-24 horas. Os suínos controles não apresentaram sinais clínicos (Quadro 1).

Os valores séricos das enzimas AST e CPK apresentam-se nas

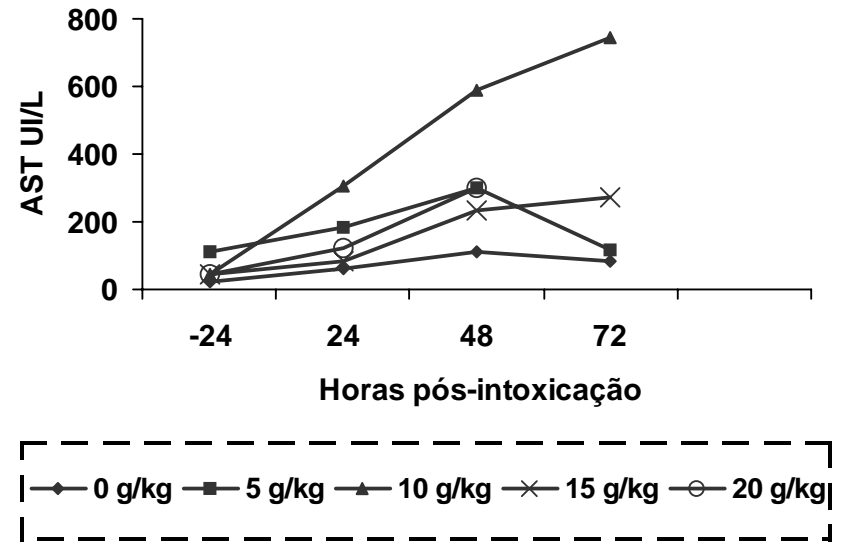

Fig.2. Evolução dos valores séricos da enzima aspartato aminotransferase (AST) em suínos intoxicados com frutos de Melia azedarach.

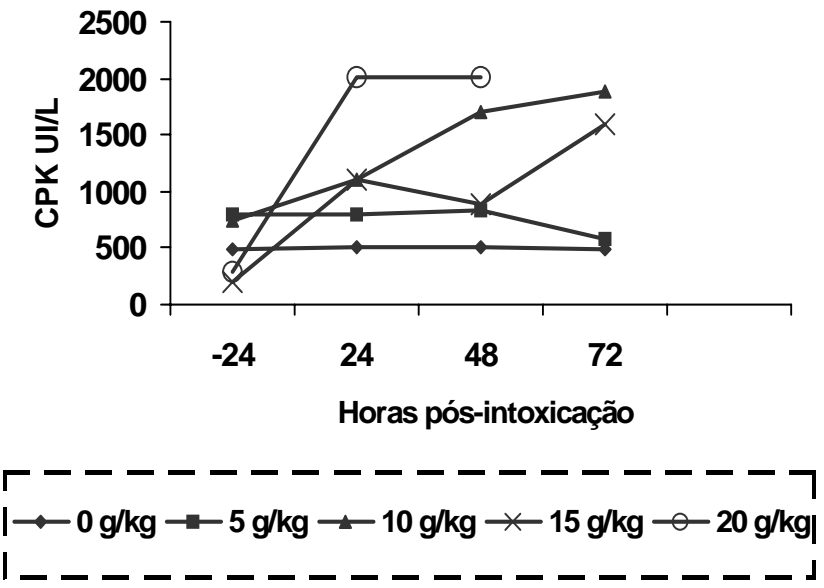

Fig.3. Evolução dos valores séricos da enzima creatinina fosfoquinase

(CPK) em suínos intoxicados com frutos de Melia azedarach.

Quadro 2. Correlação entre as doses de frutos de Melia azedarach administradas a suínos e os valores enzimáticos de AST e CPK

\begin{tabular}{ccc}
\hline $\begin{array}{c}\text { Doses } \\
\mathrm{g} / \mathrm{kg}\end{array}$ & AST & CPK \\
\hline 5 & $0,218^{\mathrm{a}}$ & 0,579 \\
10 & 0,995 & 0,981 \\
15 & 0,957 & 0,901 \\
20 & 0,965 & 0,870 \\
\hline
\end{tabular}

${ }^{\mathrm{a} O s}$ números indicam o valor de $r$

Figuras 2 e 3, respectivamente. Observou-se diferença significativa nos níveis de AST somente entre os valores registrados nos suínos que receberam doses de $10 \mathrm{~g} / \mathrm{kg}$, as 48 e 72 horas, com relação ao restante dos animais. Não se observaram diferencias significativas nos níveis de AST entre grupos. A CPK mostrou uma elevação significativa às 24 horas nos suínos dosados com $20 \mathrm{~g} / \mathrm{kg}$. A enzima AST mostrou correlação com a dose para to- 


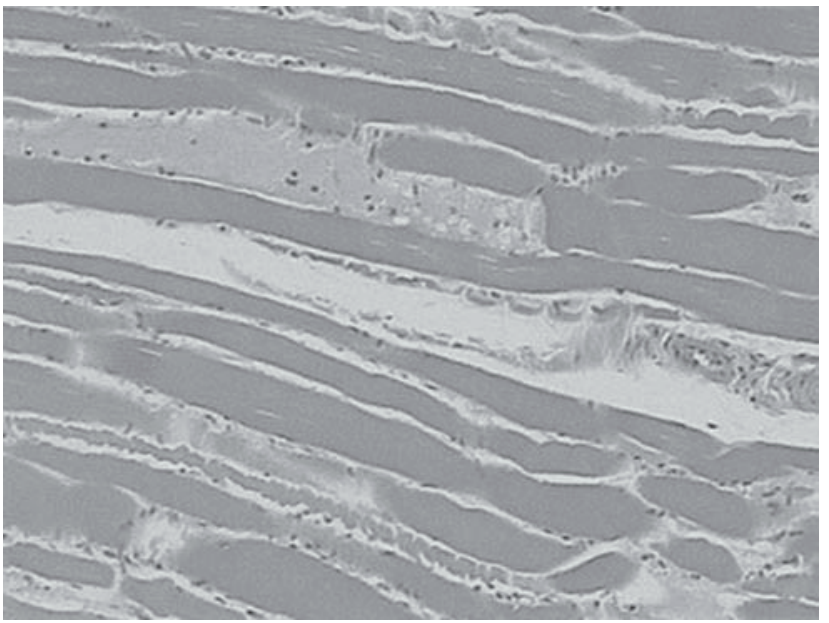

Fig.4. Corte longitudinal do músculo tríceps do Suíno 8, intoxicado com $20 \mathrm{~g} / \mathrm{kg}$ de frutos de Melia azedarach. Observam-se degeneração e necrose segmentar com hialinização de fibras, que se apresentam de cor rosa claro, com aspecto granular, e com restos nucleares nas margens da fibra. HE, obj.10.

das as doses recebidas, com exceção a dose de $5 \mathrm{~g} / \mathrm{kg}$ (Quadro 2). Todas as doses de frutos recebidas pelos suínos estiveram correlacionadas com os valores enzimáticos da CPK (Quadro 2).

As alterações macroscópicas, nos dois suínos que morreram, consistiram em congestão do intestino delgado e da mucosa do estômago.

As alterações histológicas observadas foram: degeneração gordurosa hepática e renal; congestão e edema pulmonar; severa congestão do baço; congestão e necrose da mucosa gástrica e do intestino delgado; e discreta a moderada necrose de linfócitos nos linfonodos e tecido linfático do intestino.

Nos músculos esqueléticos estudados observou-se degeneração hialina, fragmentação e necrose de fibras (Fig.4). Nas fibras necróticas observava-se material de aspecto granular, ausência de núcleos e restos nucleares nas margens da fibra. Infiltração de macrófagos e de células satélites de aspecto vesicular foi freqüientemente observada nos fragmentos necróticos.

As alterações ultraestruturais observadas nos músculos esqueléticos do Suíno $8(20 \mathrm{~g} / \mathrm{kg})$ caracterizaram-se pela presença de mitocôndrias dilatadas com cavidades matriciais eletrolúcidas e distorsões das crestas. Outras mitocôndrias apresentavam esférulas eletrodensas na matriz mitocondrial. Conservou-se a disposição das miofibrilas, retículo sarcoplásmico e túbulos T.

\section{DISCUSSÃO}

Em nossos experimentos, todas as doses de frutos de Melia azedarach utilizadas $(5-20 \mathrm{~g} / \mathrm{kg})$ produziram sinais clínicos e $20 \mathrm{~g} /$ $\mathrm{kg}$ foram letais, causando a morte 1-3 horas após os primeiros sinais. Os animais que sobreviveram recuperaram-se dentro de 24 horas. Esses dados são similares aos descritos por outros autores (Kingsbury 1964, Everist 1974, Kwatra et al. 1974, Oelrichs et al. 1985).

Segundo Oelrichs et al. (1983), a diarréia ocorre quando os animais ingerem doses não letais de frutos e eles se recuperam em 2-3 dias. Doses de $5 \mathrm{~g} / \mathrm{kg}$ causaram diarréia nos suínos de nossos experimentos, e os animais se recuperaram dentro de 24 h. Doses não letais de folhas de cinamomo causam, também, diarréia em bovinos, ocorrendo recuperação dos animais em 34 dias. Por outro lado, a ingestão de doses letais de folhas, produz fezes secas com sangue (Méndez et al. 2002a).

Os sinais nervosos observados neste trabalho nos suínos intoxicados com frutos de $M$. azedarachs são similares aos descritos por outros autores (Kwatra et al.1974, Oelrichs et al. 1985, Hare 1998).

Na patologia clínica houve aumento dos níveis séricos das enzimas CPK e AST, devido, pelo menos em parte, a mionecrose. No entanto, não ocorreu mioglobinúria, o que pode ser explicado pelo curso clínico agudo. Em bovinos e ovinos intoxicados por M. azedarach, também não se observa mioglobinúria (Méndez 2003, (Méndez et al. 2002a,b).

Os resultados do estudo histopatológico mostraram alterações degenerativas moderadas no fígado e no rim; necrose discreta a moderada de linfócitos no tecido linfático; congestão e necrose da mucosa gástrica e do intestino delgado; e degeneração e necrose dos músculos esqueléticos. Degeneração gordurosa e hiperemia do fígado e rim são alterações descritas em animais intoxicados que sobrevivem alguns dias (Kingsbury 1964, Everist 1974, Oelrichs et al. 1985). Em bovinos intoxicados com M. azedarach observa-se degeneração e necrose em órgãos do sistema digestivo, necrose do tecido linfático, necrose dos músculos esqueléticos e moderadas alterações no fígado e rim (Méndez et al. 2002a,b). Em ovinos, as lesões são similares, não se observando, no entanto, necrose do sistema digestivo (Méndez 2003). Em suínos descreve-se necrose da mucosa gástrica como a principal alteração. Outras lesões incluem cilindros hialinos nos túbulos renais e necrose de linfócitos em linfonodos e no baço (Kwatra et al. 1974, Oelrichs et al. 1985).

A degeneração e necrose das fibras musculares esqueléticas observadas nos suínos foram similares às descritas em ratos alimentados com folhas de M. azedarach (Bahri et al. 1992) e às descritas em ovinos (Méndez 2003) e em bovinos, tanto na intoxicação pelos frutos (Méndez et al. 2002b), quanto pelas folhas (Méndez et al. 2002a). Na intoxicação de suínos com frutos de cinamomo são descritos espasmos neuromusculares (Oelrichs et al. 1983, 1985), mas não se descrevem lesões nos músculos esqueléticos.

Pelos resultados obtidos neste trabalho é evidente que no estudo histológico, a comprovação das lesões de necrose do sistema digestivo e linfonodos mesentéricos e necrose segmentar muscular são de utilidade para o diagnóstico da intoxicação.

Diferentes substâncias têm sido isoladas e caracterizadas como princípios tóxicos de M. azedarach. Para alguns autores a toxina está na polpa do fruto, e é um alcalóide de estrutura química desconhecida; outros isolaram saponinas com propriedades hemolizantes (Morrison \& Grant 1932, Huang et al. 1995) e vários limonoides potencialmente tóxicos (Ahn et al. 1994, Nakatani et al. 1994, Huang et al. 1995). As análises realizadas por Carratala (1939) revelaram a presença de um alcalóide, a azaridina, uma resina, tanino e ácidos meliotânico e benzóico. Oelrichs et al. (1983) isolaram e identificaram tetranortriterpenos 
da classe limonoides, denominadas meliatoxinas A1, A2, B1 e B2, como princípios tóxicos dos frutos de $M$. azedarach (Oelrichs et al. 1983, 1985). Esses autores sugerem que essas substâncias seriam responsáveis pelos sinais nervosos agudos e morte em suínos e uma outra substância, não identificada ainda, seria responsável pelos sinais digestivos. 0 mecanismo de ação de essas substâncias não é conhecido (Oelrichs et al. 1983, 1985).

Os resultados do estudo clínico-patológico e enzimático, em suínos intoxicados com frutos de M. azedarach, obtidos neste trabalho, assim como os dados da intoxicação em bovinos e ovinos (Méndez 2003), mostram que a intoxicação por essa planta afeta vários órgãos e sistemas. Os resultados das análises bioquímicas e o estudo da patologia não permitiram, no entanto, determinar a causa da morte dos animais. $\mathrm{O}$ conhecimento do mecanismo de ação e da bioquímica das sustâncias tóxicas responsáveis pela intoxicação é muito importante para elucidar a patogenia da doença, assim como talvez desenvolver um antídoto.

Agradecimentos.- Trabalho apoiado pelo CNPq, FAPERGS e pelo Programa de Apoio a Núcleos de Excelência (Pronex).

\section{REFERÊNCIAS}

Ahn J., Choi S. \& Lee C. 1994. Cytotoxic limonoids from Melia azedarach var. japonica. Phytochemistry 36:1493-1496.

Bahri S., Sani Y. \& Hooper P.T. 1992. Myodegeneration in rats fed Melia azedarach. Aust. Vet. J. 69:33.

Carratala R.E. 1939. Intoxicación mortal por frutos de Melia azedarach L. (paraíso vegetal). Revta Asoc. Med. Argent. 53:338-340.

Everist S.L. 1974. Poisonous Plants of Australia. Angus and Roberts Pty, Sydney, Australia, p.368-369.

Hare W.R. 1998. Chinaberry (Melia azedarach) poisoning in animals, p.514516. In: Garland T. \& Barr A.C. (ed.) Toxic Plants and Other Natural Toxicants. CAB International, Wallingford.

Hare W.R., Schutzman H., Lee B.R. \& Knight M.W. 1997. Chinaberry poisoning in two dogs. J. Am. Vet. Med. Assoc. 210:1638-1640.
Hothi D.S., Singh B.D.S., Kwatra M.S. \& Chawla R.S. 1976. A note on the comparative toxicity of Melia azedarach (DHREK) berries to piglets, buffalocalves and fowls. J. Res. Punjab Agric. University 13:232-234.

Huang R.C.H., Okamura H., Iwagawa T. \& Tadera K. 1995. Azedarachin C, a limonoid antifeedant from Melia azedarach. Phytochemistry 38:593-594.

Hurst E. 1942. Poison Plants of New South Wales. Plants Committee, Sydney, NSW, Australia, p.214-218.

Kingsbury J.M. 1964. Poisonous Plants of the United States and Canada. Prentice-Hall Inc., Englewood Cliffs, NJ, p.206-208.

Kwatra M.S., Singh B.D.S., Hothi D.S. \& Dhingra P.N. 1974. Poisoning by Melia azedarach in pigs. Vet. Rec. 94:421.

Méndez M.C. 2003. Estudios clínicos, antomopatológicos y enzimáticos sobre la toxicidad de hojas y frutos de Melia azedarach para bovinos, ovinos y cerdos. Tesis, Universidad Nacional de La Plata, La Plata, Argentina. $67 \mathrm{p}$.

Méndez M.C., Aragão M., Elias F., Riet-Correa F. \& Gimeno E.J. 2002a. Experimental intoxication by leaves of Melia azedarach in cattle. Pesq. Vet. Bras. 22:19-24.

Méndez M.C., Elias F., Aragão M., Gimeno E.J. \& Riet-Correa F. 2002b. Experimental intoxication by fruits of Melia azedarach in cattle. Vet. Human. Toxicol. 44:145-148.

Morrison F.R.A. \& Grant R.A. 1932. A contribution to the chemistry of the fruit obtained from the white cedar tree (Melia azedarach L. var. australasica CDC; syn. Melia australasica A.Juss.) growing in New South Wales, with notes on its reputed toxicity. J. Proc. R. Soc. N. S. Wales 65:153.

Nakatani M., Huang R.C.H., Okamura H., Naoki H. \& Iwagawa T. 1994. Limonoid antifeedants from Chinese Melia azedarach. Phytochemistry 36:39-41.

Oelrichs P.B., Hill M.W., Vallely P.J., Macleod J.K. \& Molinski T.F. 1983. Toxic tetranortriterpenes of the fruit of Melia azedarach. Phytochemistry 22(2):531-534.

Oelrichs P.B., Hill M.W., Vallely P.J., Macleod J.K. \& Molinski T.F. 1985. The chemistry and pathology of meliatoxins A and B constituents from the fruit of Melia azedarach L. var. australasica, p.387-394. In: Seawright A.A., Hegarty M.P. \& James L.F. (ed.) Plant Toxicology. Queensland Poisonous Committee, Yeerongpilly, Australia.

Timm C. \& Riet-Correa F. 1997. Plantas tóxicas para suínos. Ciência Rural, Santa Maria, 27(3):521-528. 